\title{
Interference Aware \& SINR Estimation in Femtocell Networks
}

\author{
Kanak Raj Chaudhary ${ }^{1}$, Deepesh Rawat ${ }^{2}$, Eisha Madwal ${ }^{3}$ \\ 1. Mtech (Digital Communication), BTKIT,Dwarahat Uttarakhand, India) \\ ${ }_{2}$ Mtech (Digital Communication), BTKIT,Dwarahat, Uttarakhand, India) \\ 3, Mtech (Communication Engineering) GEU,Dheradun, Uttarakhand, India)
}

\begin{abstract}
In wireless communication two main limitations are capacity and range. In the areas of high population density cellular service is far superior compared to scarcely populated areas. The initial cellular systems were designed for a single application, that is only for voice, but today with the advent of third-generation $(3 G)$ cellular systems, users expect not only good quality of voice but also many other features such as uninterrupted voice calls, clear video images and faster internet facilities. Data traffic is usually bursty in nature and requires more bandwidth than traditional voice service. $3 G$ suffers from a limitation that it provides inadequate indoor signal penetration, which leads to poor coverage in the indoor environment where users spend most of their time. These characteristics indicate that future cellular wireless systems must be designed in a different way, hence the motivation to move towards smaller cells that operate in a licensed spectrum but are privately owned. Femtocells provide a good solution to overcome indoor coverage problems and also to deal with the traffic within Macro cells. Femtocells provide reliable and high quality of service to all customers. In this paper author has proposed the interference aware \& SINR estimation of femtocell for different distance.
\end{abstract}

Keywords - Femtocell, HeNB, LC-RRM Techniquem Microcell

\section{INTRODUCTION}

Capacity demands of modern mobile telecommunication networks are increasing year by year. People all over the world are using not only more voice call services but also an increasing amount of data services with their cell phones. Many of these services, including Web surfing, emails, video streaming, downloading video calls and banking require high speed connections and generate large amounts of data traffic to the network. The customer expectations are continuously rising and soon the mobile terminals will have to achieve the same bitrates as the current fixed internet connections. Femtocells offer a different approach to all these problems. Femto is a factor that denotes one thousandth of nano. Femtocells are very small, and have low cost base stations and their maximum allowed transmit power level is low. Femtocells are quite smaller than nanocells but the bigger difference is not the size of the cell. Like any other private companies, the mobile operators are always interested in increasing their profits. Increasing the total network capacity by deploying femtocells will save in future macrocell investments. Users that have installed a femtocell if satisfied with the service are unlikely to change their subscription to the competitor. Operators will probably also offer special femtocell billing schemes which will furthermore help in retaining customers. Deploying femtocells, on the other hand, is not free of charge either. Even though the devices are relatively low-cost the operators will have to spend large amounts of money on device distribution and marketing. In addition, many users will need helpdesk phone lines or some other means of support in installing the device [1].

\section{OPERATION OF FEMTOCELL}

A broadband internet connection is a prerequisite for connecting a femtocell. The femtocell encryped all voice calls and data sent or received by the mobile phone. This makes it impossible for an external user to break into a user's home network. For a standard $3 \mathrm{G}$ cellular phone, the femtocell appears as another cell site or macrocell, hence communicating with it as it would with a macrocell, when the mobile phone is used outdoors. Since femtocells operate at very low radio power levels, battery life is high. Also call quality is excellent, when the distance between the femtocell and the mobile handset is short. The mobile operator's data switch and telephone switch communicate with the femtocell gateway in the same way as for other mobile calls. Therefore, all services including call diversion, phone numbers, and voicemail etc. all operate in exactly the same way and appear the same to the end user. The connection between the femtocell gateway and the femtocell is encrypted using IPSec, which prevents interception. There is also authentication when the femtocell is installed for the first time to ensure that the access point is a valid one. Inside the femtocell there are the complete workings of a mobile phone base station. Some additional functions are also included, such as the RNC (Radio Network Controller) processing, which would normally reside at the mobile switching center. Some femtocells also include core network element so that data sessions can be managed locally without needing to flow back through the operator's switching centers. 


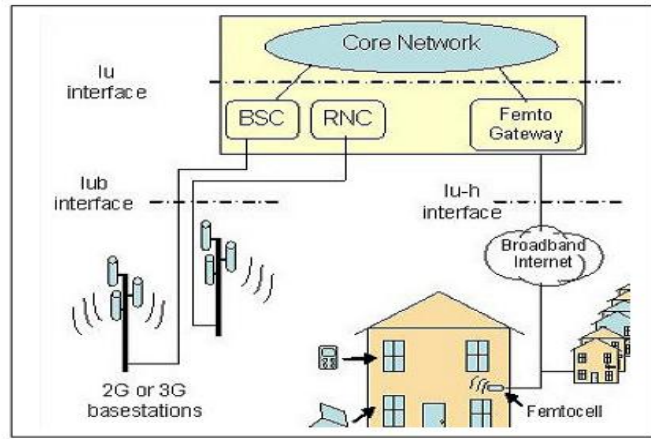

Fig. 1: Femtocell deployment

In [4] interference mitigation between neighboring femtocells and between the femtocell and macrocell is considered to be one of the major challenges in femtocell networks because femtocells share the same licensed frequency spectrum with macrocell. Further, overly concerned with convention radio resource management techniques for hierarchical cellular system is not suitable for femtocell networks since the position of the femtocells is random depending on the users' service requirement. In this, we provide a survey on the different state-of-the-art approaches for interference and resource management in orthogonal frequency-division multiple access (OFDMA) based femtocell networks. A qualitative comparison among their different approaches is provided. To this end, an open challenges in designing interference management schemes for OFDMA femtocell networks.

\section{TeChNical Challenges In Femtocell DePloyment}

The mass deployment of femtocells gives rise to several technical challenges. One of the major challenges is interference management between neighboring femtocells and between femtocell and macrocell. There are two types of interferences that occur in a two-tier femtocell network architecture (i.e., a central macrocell is underlaid/overlaid with 3G/OFDMA femtocells, respectively) are as follows:

\subsection{Co-tier interference:}

This type of interference occurs among network elements that belong to the same tier in the network. In femtocell network, co-tier interference occurs between neighboring femtocells. For example, a femtocell UEs (aggressor) causes uplink co-tier interference to the neighboring femtocell base stations (victims). On the other hand, a femtocell base station acts as a source of downlink co-tier interference to the neighboring femtocell UEs. However, in 3G/OFDMA systems, the co-tier uplink or downlink interference occurs only when the aggressor (or the source of interference) and the victim use the same sub-channels. Therefore, proper allocation of subchannels is required in 3G/OFDMA-based femtocell networks to mitigate co-tier interference.

\subsection{Cross-tier interference:}

This type of interference occurs among network elements that belong to the different tiers of the network, i.e., interference between femtocells and macrocells. For example, femtocell UEs \& macrocell UEs (also referred to as MUEs) and act as a source of uplink cross-tier interference to the serving and the nearby femtocells \& macrocell base station, respectively. On the other hand, the serving macrocell base station and femtocells cause downlink cross-tier interference to the femtocell UEs and nearby macrocell UEs, respectively. Again, in OFDMA-based femtocell networks, interference occurs only when the same sub-channels are used by the aggressor and the victim i.e (cross-tier uplink or downlink). Femtocells are spread over the existing macrocell network and share the same frequency spectrum with macrocells. Due to spectral scarcity, the macrocells and femtocells have to reuse the total allocated frequency band partially or totally which leads to cochannel or cross-tier interference. At the same time, femtocells should occupy as little bandwidth as possible that leads to co-tier interference, in order to guarantee the required QoS to the macrocell users. 


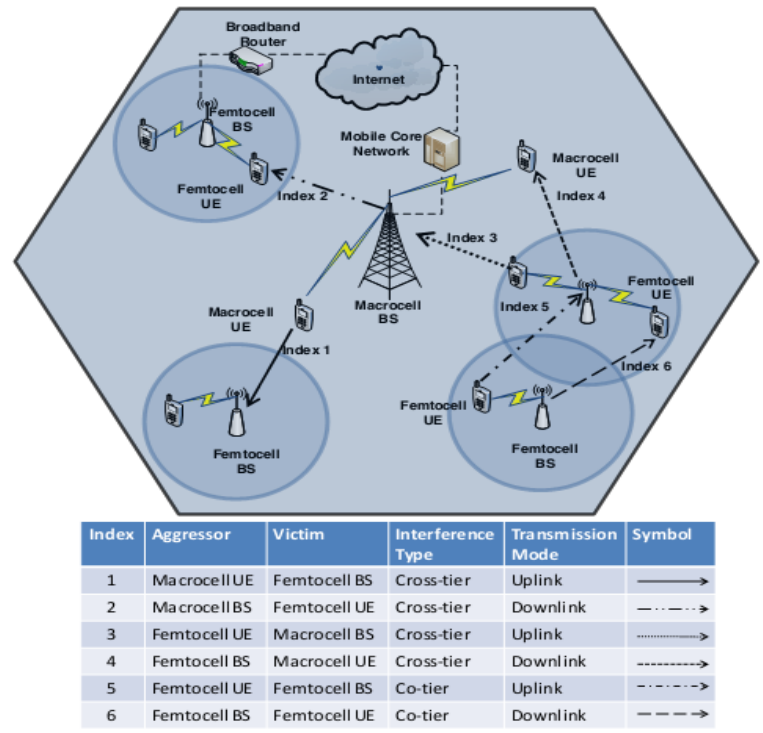

Fig. 2: Interference scenarios in OFDMA-based femtocell networks.

As a result, the throughput of the network would decrease substantially because of such co-tier and cross-tier interference. In addition, severe interference may causes to "Dead zones", i.e., areas where the QoS degrades significantly. These dead zones are created due to asymmetric level of transmission power within the network and the distance between macrocell base station and macrocell UE. For example, due to co-channel interference a macrocell UE located at a cell edge and transmitting at a high power will create a dead zone to the nearby femtocell uplink transmission. On the other hand, in the downlink transmission, a cell edge macrocell UE may experience severe co-channel interference from the nearby femtocells, due to high path-loss and shadowing effect. Thus, it is essential to adopt an effective and robust interference management scheme that would mitigate the co-tier interference and reduce the cross-tier interference considerably in order to enhance the throughput of the overall network.

In OFDMA-based femtocell networks, orthogonal sub-carriers can be assigned to femtocells and macrocells, due to the flexibility in spectrum allocation. This gives OFDMA-based femtocells an edge over CDMA systems in terms of utilizing the frequency spectrum resources efficiently. Fig. 2 illustrates all possible interference scenarios in an OFDMA-based femtocell network. If an effective interference management scheme can be adopted, then the co-tier interference can be mitigated and the cross-tier interference can be reduced which would enhance the throughput of the overall network.

\section{INTERFERENCE MANAgEMENT APPROACH USING FFr \& RESOURCE PARTITIONING}

The basic mechanism of this method divides the entire frequency spectrum into several sub-bands. Afterwards, each sub-band is differently assigned to each macrocell or sub-area of the macrocell. Since the resource for HeNB and MeNB is not overlapped, interference between MeNB and HeNB can be minimised. The authors proposed a frequency sharing mechanism that uses frequency reuse coupled with pilot sensing to reduce cross-tier/co-channel interference between femtocells and macrocell. In this scheme, FFR of 3 or above is applied to the macrocell. When a HeNB is turned on, it detect the pilot signals from the MeNB and discards the sub- band with the maximum strenght received signal power, and thus uses the rest of the frequency sub-bands resulting in an increased SINR for macrocell UEs. The overall network outturn is enhanced by adopting highorder modulation schemes.

Fig. 3(b) illustrates the allocation of frequency sub-bands within the macrocell sub-areas. The sub-band $\mathrm{A}$ is used in the center zone $(\mathrm{C} 1, \mathrm{C} 2$, and $\mathrm{C} 3)$, and sub-bands $\mathrm{B}, \mathrm{C}$, and $\mathrm{D}$ are used in regions $\mathrm{X} 1, \mathrm{X} 2$, and $\mathrm{X} 3$, respectively. When a HeNB is turned on, it senses the neighboring MeNB signals and compares the Received Signal Strength Indication (RSSI) values for the sub-bands, and chooses the sub-bands that are not used in the macrocell sub-area. If the HeNB is located in the center zone then it excludes the sub-band that is used in the center zone as well as the one that is used by the macrocell in the edge region of the current sector. For example, if a HeNB is located in edge region X1, then it would obviate sub-band B which is used by macrocell UEs, and select sub-band A, C, or D. However, if a HeNB is located in center zone C1, then it avoids sub-band A and at the same time sub-band B since the RSSI for this sub-band is comparatively higher for that HeNB. In this way, this scheme abates co-tier and cross-tier interference. Simulation results show that, the expedient offers 
throughput gains of $27 \%$ and $47 \%$ on average, when compared with the FFR-3 scheme (with no center zone) and a scheme with no FFR, respectively.

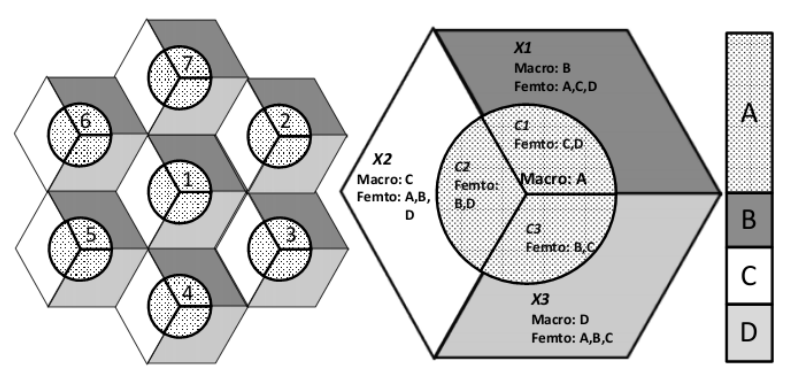

(a)

(b)

Fig. 3: Interference management scheme using FFR

Femtocells are a solution that helps to reduce the capital and operational expenditure of a mobile network while enhancing system coverage and capacity. However, the dodge of interference is still an issue that needs to be addressed to successfully deploy a femtocell tier over existing macrocell networks. Moreover, interference is strongly clinging on the type of access control, which adjudges if a given user can or cannot connect to the femtocell. In [5], the existing access methods for femtocells together with their benefits and drawbacks are explained. Different approaches have been proposed for access(Fig. 4):

Closed access: Only a subset of users, defined by the femtocell owner, can connect to the femtocell. This model is referred to as closed subscriber group (CSG) by the Third Generation Partnership Project (3GPP).

Open access: All customers of the operator have the right to make use of any femtocell.

Hybrid access: A limited amount of the femtocell resources are available to all users, while the rest are operated in a CSG manner.

When the access method blocks the use of femtocell resources to a subset of the users within its coverage area, a new set of interfering signals is implicitly defined in such area. Hence, the deployment of CSG femtocells makes the problem of interference mitigation even more complex. Contrarily, the deployment of open FAPs would solve this issue, but bring security and sharing concerns to the customer. Further-more, when users move across areas with large numbers of open FAPs, the number of handovers and thus the signaling in the network increases. Finally, hybrid access techniques can be seen as a trade-off between open and closed approaches. However, the number of shared resources must be carefully tuned to avoid a large impact on the quality of service of the femtocell customers.

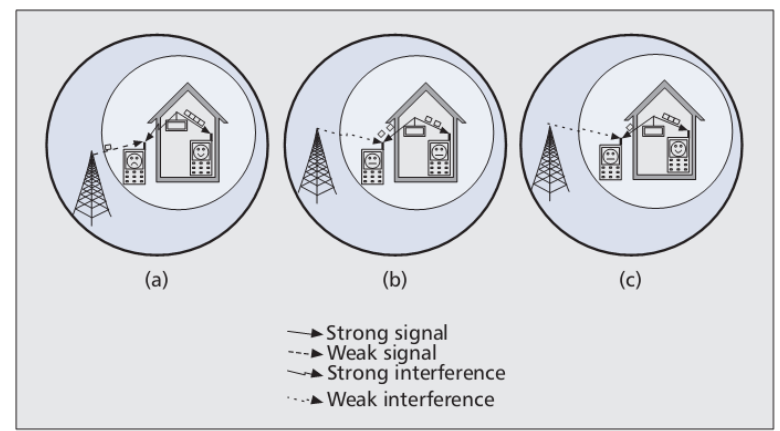

Fig. 4: Access methods: a) closed subscribers group (CSG); b) open access; c) hybrid access.

In [6] the author has proposed the use of fractional frequency reuse (FFR) to mitigate inter-femtocell interference in multi-femtocell environments. We consider a simple femtocell environment comprising two femtocells as illustrated in Fig. 4. Let femtocell $\mathrm{k}$ and 1 be the target and interfering femtocell with coverage radius rk and r, respectively. For the ease of description, define orthogonal area (OA), $\Psi_{\mathrm{k}, 1}$, by the portion of the coverage area of femtocell $\mathrm{k}$, where the average signal-to-interference power ratio(SIR) associated with the interference from femtocell 1 is lower than a given threshold level $\lambda_{\text {th }}$. To provide desired cell edge spectral efficacy, it may need to apportion frequency resource to users in OA in an orthogonal manner. The shape of the border where the average SIR is equal to $\lambda_{\text {th }}$ is shown in Fig.5 as a circle with radius $R_{0}$. Define Ratio of Orthogonal Area (ROA), $\Omega \mathrm{k}, \mathrm{l}$, by the ratio of the OA to the entire femtocell coverage with radius $\mathrm{r}$ k (i.e., $\Omega_{\mathrm{k}, \mathrm{l}}=\Psi_{\mathrm{k}, \mathrm{l}} / \pi \mathrm{r}^{2} \mathrm{k}$ ). If users are uniformly distributed in femtocell $\mathrm{k}$, the probability of users to be located in OA $\Psi_{\mathrm{k}, \mathrm{l}}$ is $\Omega_{\mathrm{k}, \mathrm{I}}$ Note that the OA and ROA are a function of the distance. With the uploaded OAM information that 
includes the distance between femtocells, the femto gateway can successfully allocate frequency resource to femtocells by estimating mutual IFI.

In [7] the authors have proposed a way of mitigation of interference, which is a distributed and selforganizing femtocell management architecture, called the Complementary TRi-control Loops (CTRL), that consists of three control loops to determine maximum transmit power of femtocell users based on the fed-back macrocell load margin for protection of the macrocell uplink communications; (2) target signal to interference plus noise ratios (SINRs) of femtocell users to reach a Nash equilibrium; and (3) instantaneous transmit power of femtocell users to achieve the target SINRs against bursty interference from other nearby users.

\section{Simulation Result}

We consider a network of $(300 \mathrm{X} 300) \mathrm{Km}$, in which one macro station , which is situated at center as see in (fig 5) \& many macro users place around it. Similarly, there are many femto Stations are situated in this network which are access by their femto users.

We evaluate the path loss for macro \& femto network separately.Path Loss for macro represented as below $\mathrm{PL}_{\text {macro }}=10^{\left(\mathrm{PL}_{-} \mathrm{cst}+\mathrm{PL} \_ \text {gain*log }\right.}{ }_{10}$ (distance) $\left./ 10\right)$

PLfemto $=100^{(\text {PL_cst+PL_gain*log10(distance) }+0.7 * \text { distance+wall_penetration }) / 10)}$

PL_Cst $t_{(\mathrm{femto})}=137.74 \quad \& \quad$ PL_Gain ${ }_{(\mathrm{femto})}=30$

while

$\mathrm{PL} \_C s t_{(\text {macro })}=128.1 \quad \& \quad$ PL_Gain (macro) $=37.6$

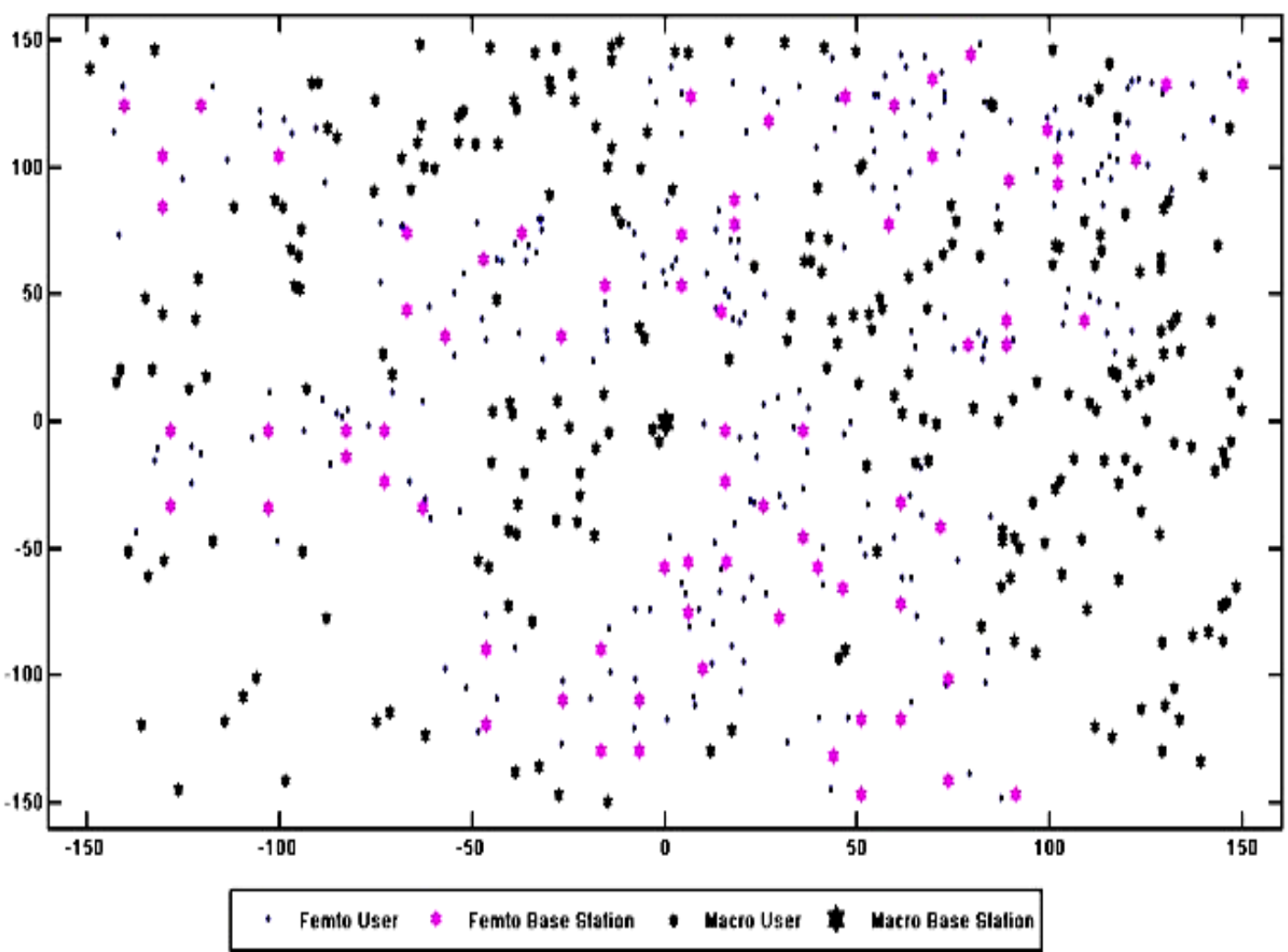

Fig. 5: Macro \& Femto Stations \& Users Network Graph 


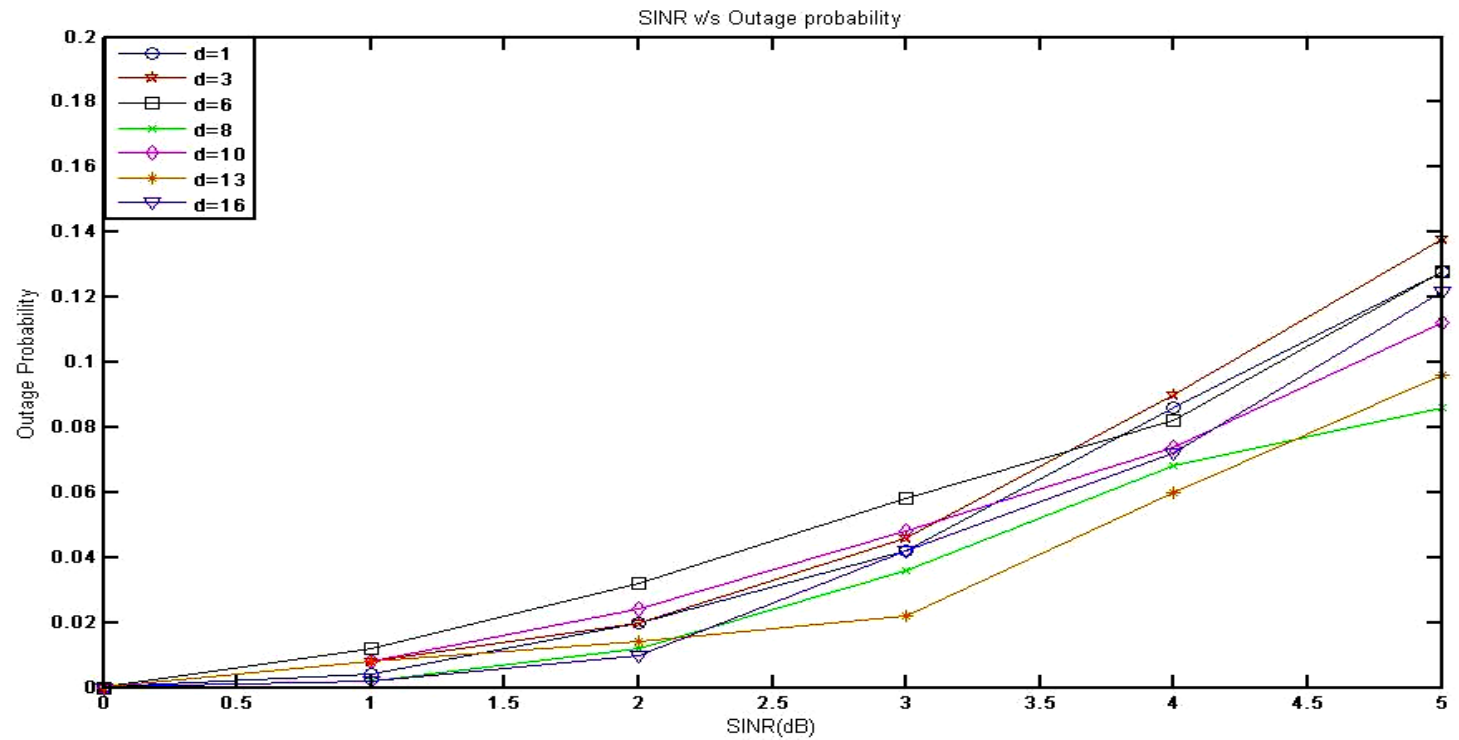

Fig. 6: SINR Vs Outage probability Graph

SINR estimation of femtocell user using a Rayleigh channel. The channel we use, it is frequency-flat Rayleigh channel with no Doppler Shift. It is static channel. The sample time of the input signal is irrelevant for frequency-flat static channel.We see the SINR Vs outage Probability graph of for seven different distances, shown in fig 6.

\section{CONCLUSION}

In this paper, we proposed the interference aware \& SINR estimation of femtocell for different distance as we seen in graph. We in graph that at distance $\mathrm{d}=10$ the outage probability is low, which is true according to standard evaluations. In continuation to this study, we are working on the SINR estimation for many static \& dynamic channel for MeNB \& HeNB, And other pratical model providing user mobility with dynamic deployment of $\mathrm{HeNb}$ etc. More studies in this area are being investigated further.

\section{REFERENCES}

[1]. MikkoJärvinen, "Femtocell Deployment in $3^{\text {rd }}$ Generation Networks", Master's Thesis, HELSINKI UNIVERSITY OF TECHNOLOGY, 2009

[2]. Vikram Chandrasekhar and Jeffrey G. Andrews, The University of Texas at Austin, Alan Gatherer, Texas Instrument, "Femtocell Networks: A Survey", IEEE Communications Magazine, 2008

[3]. KhaledElleithy and VarunRao , "Femto Cells: Current Status and Future Directions", International Journal of Next-Generation Networks (IJNGN) Vol.3, No.1, March 2011

[4]. Nazmus Saquib, Ekram Hossain, Long Bao Le, and Dong In Kim, "Interference Management in OFDMA Femtocell Networks: Issues and Approaches", 2011

[5]. Guillaume de la Roche, Alvaro Valcarce, David López-Pérez, and Jie Zhang, "Access Control Mechanisms for Femtocells", IEEE Communications Magazine, January 2010

[6]. Heui-Chang Lee, Dong-Chan Oh, and Yong-Hwan Lee "Mitigation of Inter-Femtocell Interference with Adaptive Fractional Frequency Reuse Orthogonal Area and Ratio of Orthogonal Area", IEEE, 2010

[7]. Ji-Hoon Yun, Member, IEEE, and Kang G. Shin, Fellow, IEEE “Adaptive Interference Management of OFDMA Femtocells for CoChannel Deployment", IEEE JOURNAL ON SELECTED AREAS IN COMM UNICATIONS, VOL. 29, NO. 6, JUNE 2011

[8]. Francesco Pantisano, Mehdi Bennis, WalidSaad, M'erouaneDebbah, "Cooperative Interference Alignment in Femtocell Networks", IEEE, 2011

[9]. Ju Yong Lee, Member, IEEE, Sueng Jae Bae, Student Member, IEEE, Young Min Kwon, and Min Young Chung," Interference Analysis for Femtocell Deployment in OFDMA Systems Based on Fractional Frequency Reuse Member", IEEE COMMUNICATIONS LETTERS, VOL. 15, NO. 4, APRIL 2011

[10]. Yu-Shan Liang, Wei-Ho Chung, Member, IEEE, Guo-Kai Ni, Ing-Yi Chen,Hongke Zhang, and Sy-Yen Kuo,Fellow, "Resource Allocation with Interference Avoidance in OFDMA Femtocell Networks", IEEE TRANSACTIONS ON VEHICULAR TECHNOLOGY, VOL. 61, NO. 5, JUNE 2012

[11]. Mustafa E. Şahin, Ismail Guvenc, Moo-RyongJeong, and Hüseyin Arslan, "Handling CCI and ICI in OFDMA Femtocell Networks Through Frequency Scheduling", IEEE

[12]. RajarshiMahapatra and Emilio CalvaneseStrinati, "Radio Resource Management in Femtocell Downlink Exploiting Location Information", 2011 